\title{
A PROBLEM ON INVERSE MAPPING SYSTEMS ${ }^{1}$
}

\section{LEON HENKIN ${ }^{2}$}

Let $A$ be a set of indices $\alpha, \beta, \cdots$ directed by an ordering relation $<$; that is, for each $\alpha, \beta$ there is a $\gamma$ such that $\alpha<\gamma$ and $\beta<\gamma$. By an inverse-mapping-onto-system of type $\mathrm{A}$ is meant a family of nonempty disjoint sets $M_{\alpha}$ together with functions $\Pi_{\alpha}^{\beta}$, for each $\alpha<\beta$, mapping $M_{\beta}$ onto $M_{\alpha}$. It is required that these functions be such that if $\alpha<\beta<\gamma$ then $\Pi_{\alpha}^{\beta}\left(\Pi_{\beta}^{\gamma} x\right)=\Pi_{\alpha}^{\gamma} x$ for each $x \in M_{\gamma}$. The inverse limit of such a system consists of those functions $f$ defined over $A$ such that $f(\alpha)=x_{\alpha} \in M_{\alpha}$ and if $\alpha<\beta$ then $\Pi_{\alpha}^{\beta} x_{\beta}=x_{\alpha}$. If every inverse-mappingonto-system of type $\mathrm{A}$ has a nonempty inverse limit then the directed set $A$ is called special.

THEOREM. The necessary and sufficient condition for $A$ to be special is that it possess either a maximal element or a simple cofinal sequence.

To show sufficiency in the case where $A$ has a maximal element is trivial. If $\alpha_{1}<\alpha_{2}<\ldots$ is a simple cofinal sequence in $A$, an element of the inverse limit may be constructed by choosing $x_{\alpha_{1}}$ arbitrarily in $M_{\alpha_{1}}$, and selecting $x_{\alpha_{i+1}}$ to satisfy $\Pi_{\alpha_{i}}^{\alpha_{i+1}} x_{\alpha_{i+1}}=x_{\alpha_{i}}$-possible since $\Pi_{\alpha_{i}}^{\alpha_{i+1}}$ is always onto. Finally, in case $\beta$ is not one of the $\alpha_{i}$, it is easily shown that $x_{\beta}$ is uniquely defined by the formula $x_{\beta}=\Pi_{\beta}^{\alpha_{i}} x_{\alpha_{i}}$, where any $\alpha_{i}$ such that $\beta<\alpha_{i}$ is employed. (There must be one since $\left\{\alpha_{i}\right\}$ is cofinal.) This simple argument is contained in Tukey's paper. ${ }^{1}$

To show necessity we assume that $A$ is special and construct the following inverse-mapping-onto-system of type A.

A point is a finite sequence $x=\left(\alpha_{1}, \alpha_{2}, \cdots, \alpha_{2 n-1}, \alpha_{2 n}\right)$ consisting of an even number of indices from $A$ which satisfy the following conditions:

(i) $\alpha_{1}<\alpha_{2}$,

(ii) $\alpha_{2 i-1}<\alpha_{2 i+2}$ for $0<i<n$,

(iii) $\alpha_{2 i+1}<\alpha_{2 i+2}$ and $\alpha_{2 i+1} \Varangle \alpha_{2 j+1}$ for $0 \leqq j<i<n$, where $\alpha \Varangle \beta$ holds when neither $\alpha<\beta$ nor $\alpha=\beta$.

We define index $x=\alpha_{2 n-1}$, order $x=\alpha_{2 n}$, length $x=n$.

Let $M_{\alpha}$ consist of all points of index $\alpha$. Given $\alpha<\beta$, we define the mapping $\Pi_{\alpha}^{\beta}$ by prescribing its image for an arbitrary point

Received by the editors November 26, 1948.

1 The problem treated and notation used in this paper are taken from J. W. Tukey, On denumerability in topology. The theorem proved is one of two alternative conjectures made there about the problem.

2 Frank B. Jewett Fellow. 
$x=\left(\alpha_{1}, \alpha_{2}, \cdots, \alpha_{2 n}\right)$ of $M_{\beta}$ (so that $\alpha_{2 n-1}=\beta$ ) as follows. In case $\alpha \leqq \alpha_{1}$ let $\Pi_{\alpha}^{\beta} x=\left(\alpha, \alpha_{2}\right)$; since $x$ is a point we have $\alpha_{1}<\alpha_{2}$, whence $\alpha \leqq \alpha_{1}$ implies $\alpha<\alpha_{2}$ so that $\left(\alpha, \alpha_{2}\right)$ is a point. In case $\alpha \Varangle \alpha_{1}$ we can find (since $\alpha<\beta=\alpha_{2 n-1}$ ) a least $j$ such that $\alpha \leqq \alpha_{2 j+1}$ (whence $\alpha \Varangle \alpha_{2 k+1}$ for $k<j)$. In that case we set $\Pi_{\alpha}^{\beta} x=\left(\alpha_{1}, \alpha_{2}, \cdots, \alpha_{2 j}, \alpha, \alpha_{2 j+2}\right)$, which is easily seen to satisfy conditions $\mathrm{i}$, ii, and iii if $x$ does.

These sets $M_{\alpha}$ and mappings $\Pi_{\alpha}^{\beta}$ form an inverse-mapping-ontosystem of type A. It is a simple matter to check the transitivity of the mappings: $\Pi_{\alpha}^{\beta} \Pi_{\beta}^{\gamma}=\Pi_{\alpha}^{\gamma}$ for $\alpha<\beta<\gamma$. To see that $\Pi_{\alpha}^{\beta}$ is onto (where $\alpha<\beta)$, let $x=\left(\alpha_{1}, \alpha_{2}, \cdots, \alpha_{2 n}\right)$ be any point of $M_{\alpha}$ (so that $\left.\alpha_{2 n-1}=\alpha\right)$. Choose $\gamma>\beta$ and consider the sequence $y=\left(\alpha_{1}, \alpha_{2}, \cdots, \alpha_{2 n}, \beta, \gamma\right)$. Since $x$ is a point and $\alpha_{2 n-1}=\alpha<\beta<\gamma$, it is only necessary to verify that $\beta \Varangle \alpha_{2 j+1}, j=0,2, \cdots, n-1$, in order to conclude that $y$ is a point. But this is certainly the case since $\beta \leqq \alpha_{2 j+1}$ and $\alpha<\beta$ imply $\alpha_{2 n-1}=\alpha<\alpha_{2 j+1}$ contrary to the fact that $x$ is a point and so satisfies iii. It is now easily seen that $y$ is in $M_{\beta}$ and $\Pi_{\alpha}^{\beta} y=x$.

Since we are assuming that $A$ is special it follows that the above inverse-mapping-onto-system has a non-empty inverse limit. Let $f$ be a function defined over $A$ such that $f(\alpha)=x_{\alpha} \in M_{\alpha}$, where $\alpha<\beta$ implies $\Pi_{\alpha}^{\beta} x_{\beta}=x_{\alpha}$. The set of orders of points $x_{\alpha}$ is cofinal in $A$, since for any $\alpha$ we have $\alpha=$ index $x_{\alpha}$ <order $x_{\alpha}$ (by $\mathrm{i}$ and iii). Hence we can prove our theorem by showing that the orders of points $x_{\alpha}$ either possess a maximal element or form a simple sequence.

To see this last fact it is only necessary to observe that if length $x_{\alpha}=$ length $x_{\beta}$ then order $x_{\alpha}=$ order $x_{\beta}$. For choose $\gamma$ so that $\alpha<\gamma$ and $\beta<\gamma$. Hence we must have $\Pi_{\alpha}^{\gamma} x_{\gamma}=x_{\alpha}$ and $\Pi_{\beta}^{\gamma} x_{\gamma}=x_{\beta}$. But from the definition of the mappings $\Pi$ it then follows that the orders of $x_{\alpha}$ and $x_{\beta}$ are certain elements $\alpha_{2 i}$ and $\alpha_{2 j}$ in the sequence $x_{\gamma}$. Since $i=$ length $x_{\alpha}=$ length $x_{\beta}=j$ we have order $x_{\alpha}=\alpha_{2 i}=\alpha_{2 j}=\operatorname{order} x_{\beta}$.

Thus if the lengths of the points $x_{\alpha}$ are unbounded there will be a simple sequence of orders $\beta$ (one for each length), which is cofinal in $A$. In the contrary case the orders form a finite cofinal subset of $A$ and so one of them must be maximal.

This completes the proof of our theorem.

Corollary I. If $A$ is special and $B$ is a cofinal subset of $A$ then $B$ is special.

Corollary II. Without the axiom of choice we can construct, for each directed set $A$, an inverse-mapping-onto-system of type A which has a nonempty inverse limit if and only if $A$ is special.

Princeton University 